\title{
Publisher Correction: Collective molecular switching in hybrid superlattices for light- modulated two-dimensional electronics
}

\author{
Marco Gobbi ${ }^{1,6}$, Sara Bonacchi ${ }^{1,7}$, Jian X. Lian², Alexandre Vercouter ${ }^{2}$, Simone Bertolazzi ${ }^{1}$, Björn Zyska ${ }^{3}$, \\ Melanie Timpel ${ }^{4}$, Roberta Tatti ${ }^{5}$, Yoann Olivier ${ }^{2}$, Stefan Hecht (1) ${ }^{3}$, Marco V. Nardi ${ }^{4}$, David Beljonne ${ }^{2}$, \\ Emanuele Orgiu (i) ${ }^{1,8} \&$ Paolo Samorì (i) ${ }^{1}$
}

Correction to: Nature Communications https://doi.org/10.1038/s41467-018-04932-z; published online 09 July 2018

The original version of this article incorrectly listed an affiliation of Sara Bonacchi as 'Present address: Institut National de la Recherche Scientifique (INRS), EMT Center, Boulevard Lionel-Boulet, Varennes, QC, J3X 1S2, 1650, Canada', instead of the correct 'Present address: Department of Chemical Sciences, University of Padua, Via Francesco Marzolo 1, 35131, Padova, Italy'. And an affiliation of Emanuele Orgiu was incorrectly listed as 'Present address: Department of Chemical Sciences, University of Padua, Via Francesco Marzolo 1, Padova, 35131, Italy', instead of the correct 'Present address: Institut National de la Recherche Scientifique (INRS), EMT Center, Boulevard Lionel-Boulet, Varennes, QC, J3X 1S2, 1650, Canada'. This has been corrected in both the PDF and HTML versions of the article.

Published online: 06 September 2018

Open Access This article is licensed under a Creative Commons Attribution 4.0 International License, which permits use, sharing, adaptation, distribution and reproduction in any medium or format, as long as you give appropriate credit to the original author(s) and the source, provide a link to the Creative Commons license, and indicate if changes were made. The images or other third party material in this article are included in the article's Creative Commons license, unless indicated otherwise in a credit line to the material. If material is not included in the article's Creative Commons license and your intended use is not permitted by statutory regulation or exceeds the permitted use, you will need to obtain permission directly from the copyright holder. To view a copy of this license, visit http://creativecommons.org/licenses/by/4.0/.
\end{abstract}

(C) The Author(s) 2018

\footnotetext{
${ }^{1}$ University of Strasbourg, CNRS, ISIS UMR 7006, 8 allée Gaspard Monge, F-67000, Strasbourg, France. ${ }^{2}$ Laboratory for Chemistry of Novel Materials, Center for Research in Molecular Electronics and Photonics, University of Mons, Place du Parc 20, 7000 Mons, Belgium. ${ }^{3}$ Department of Chemistry and IRIS Adlershof, Humboldt-Universität zu Berlin, Brook-Taylor-Str. 2, 12489 Berlin, Germany. ${ }^{4}$ Department of Industrial Engineering, University of Trento, Via Sommarive 9, 38123 Trento, Italy. ${ }^{5}$ IMEM-CNR, Institute of Materials for Electronics and Magnetism, Trento unit, Via alla Cascata 56/C, Povo, 38123 Trento, Italy. ${ }^{6}$ Present address: Centro de Fisica de Materiales (CSIC-UPV/EHU), Paseo Manuel de Lardizabal 5, E-20018 Donostia, San Sebastián, Spain. ${ }^{7}$ Present address: Department of Chemical Sciences, University of Padua, Via Francesco Marzolo 1, 35131, Padova, Italy. ${ }^{8}$ Present address: Institut National de la Recherche Scientifique (INRS), EMT Center, Boulevard Lionel-Boulet, Varennes, QC, J3X 1S2, 1650, Canada. The original article can be found online at https://doi.org/10.1038/s41467-018-04932-z. Correspondence and requests for materials should be addressed to M.G. (email: marco_gobbi001@ehu.eus) or to P.Sì. (email: samori@unistra.fr)
} 